\title{
Sequences with bounded l.c.m. of each pair of terms
}

\author{
by \\ Yong-Gao Chen (Nanjing)
}

0. Introduction. Let $A_{x}$ be a set of positive integers with the least common multiple of each pair of terms not exceeding $x$ and $\left|A_{x}\right|$ being the largest. In 1951, P. Erdös [3] proposed the following problem: what is the value of $\left|A_{x}\right|$ ? It is known that

$$
\sqrt{\frac{9}{8} x}+O(1) \leq\left|A_{x}\right| \leq \sqrt{4 x}+O(1) .
$$

For a proof one may see Erdős [4]. The problem is problem E2 and a part of problem B26 in the well known problem book [5] of Guy. Choi [1] improved the upper bound to $1.638 \sqrt{x}$, and later [2] to $1.43 \sqrt{x}$.

In number theory, it is rare to give an asymptotic formula for such a problem. In this paper an asymptotic formula for $\left|A_{x}\right|$ is given. Further, let $B_{x}$ be the union of the set of positive integers not exceeding $\sqrt{x / 2}$ and the set of even integers between $\sqrt{x / 2}$ and $\sqrt{2 x}$. It is clear that the least common multiple of each pair of terms of $B_{x}$ does not exceed $x$. We will show that $A_{x}$ is almost the same as $B_{x}$. That is,

TheOREM. We have

$$
\left|A_{x} \backslash B_{x}\right|=o(\sqrt{x})
$$

In particular,

$$
\left|A_{x}\right|=\left|B_{x}\right|+o(\sqrt{x})=\sqrt{\frac{9}{8} x}+o(\sqrt{x}) .
$$

Note. From the proof of the Theorem we will see that $o(\sqrt{x})$ can be given explicitly. By the Theorem we have

$$
\left|A_{x} \cap B_{x}\right|=\left|A_{x}\right|-\left|A_{x} \backslash B_{x}\right|=\sqrt{\frac{9}{8} x}+o(\sqrt{x})
$$

1991 Mathematics Subject Classification: Primary 11B83.

Supported by Fok Ying Tung Education Foundation and the National Natural Science Foundation of China. 
and

$$
\left|B_{x} \backslash A_{x}\right|=\left|B_{x}\right|-\left|A_{x} \cap B_{x}\right|=o(\sqrt{x}) .
$$

\section{Preliminary lemmas}

LEMma 1. Let $M$ be an integer with $M \geq 3$, and let $c_{0}, c_{1}$ and $c_{2}$ be real numbers with $c_{1} \geq c_{0}>0$. Then there exists an $x_{0}=x_{0}\left(M, c_{0}\right)$ such that if $x \geq x_{0}$ and $a_{i}, b_{i}(1 \leq i \leq t \leq M / 2)$ are integers with $\left(a_{i}, b_{i}\right)=1(1 \leq i \leq t)$ and with each prime factor of $\prod_{i=1}^{t}\left(a_{i} n+b_{i}\right)$ exceeding $M$ for any integer $n$, then there exists an integer $k \in\left(c_{1} x^{1 / 2}+c_{2}, c_{1}\left(x^{1 / 2}+x^{1 / 4}\right)+c_{2}\right)$ such that each prime factor of $\prod_{i=1}^{t}\left(a_{i} k+b_{i}\right)$ exceeds

$$
\frac{1}{6 \log M} \log x \text {. }
$$

P r o of. We employ the standard Eratosthenes-Legendre sieve. One may refer to [6], p. 31, Theorem 1.1. We take

$$
\begin{gathered}
\mathcal{A}=\left\{\prod_{i=1}^{t}\left(a_{i} k+b_{i}\right): k \in\left(c_{1} x^{1 / 2}+c_{2}, c_{1}\left(x^{1 / 2}+x^{1 / 4}\right)+c_{2}\right)\right\}, \\
\mathcal{P}=\mathcal{P}_{1}, \quad z=\frac{1}{6 \log M} \log x, \quad X=c_{1} x^{1 / 4}, \quad A_{0}=\frac{1}{2} M,
\end{gathered}
$$

$\omega(p)$ being the number of solutions of

$$
\prod_{i=1}^{t}\left(a_{i} n+b_{i}\right) \equiv 0(\bmod p) .
$$

Noting that $\overline{\mathcal{P}}=\emptyset$ we have $\left|R_{d}\right|=\left|r_{d}\right| \leq \omega(d)$ if $\mu(d) \neq 0$. By Theorem 1.1 of [6], p. 31, we have

$$
\begin{aligned}
S(\mathcal{A} ; \mathcal{P}, z) & =X W(z)+\theta\left(1+A_{0}\right)^{z} \\
& =c_{1} x^{1 / 4} \prod_{p \leq z}\left(1-\frac{\omega(p)}{p}\right)+\theta\left(1+\frac{1}{2} M\right)^{z} \\
& \geq c_{0} x^{1 / 4} \prod_{M<p \leq z}\left(1-\frac{M}{2 p}\right)-M^{z} \gg \frac{x^{1 / 4}}{(\log \log x)^{M / 2}},
\end{aligned}
$$

where $|\theta| \leq 1$ and $\gg$ depends only on $M$ and $c_{0}$. From this we obtain the assertion of Lemma 1.

Note. $a_{i}$ and $b_{i}$ may depend on $x, c_{0}, c_{1}$ and $c_{2} . x_{0}\left(M, c_{0}\right)$ can be effectively computed. For a stronger result one should use Brun's sieve. Here the conclusion is sufficient for the present paper.

LEMMA 2. Let $c_{i}(3 \leq i \leq 6)$ be nonnegative real numbers with $c_{4}>c_{3}$. Let $D$ and $M$ be integers with $|D| \leq c_{5} x^{c_{6}}$ and with each prime factor of $D$ 
exceeding

$$
\frac{1}{6 \log M} \log x .
$$

Then the number of a with $(a, D)>1, a \in\left[c_{3} x^{1 / 2}, c_{4} x^{1 / 2}\right]$ is $O(\sqrt{x} / \log \log x)$, where $O$ depends only on $M$ and $c_{i}(3 \leq i \leq 6)$.

Proof. If $D=0$, then $x \leq M^{12}$ and the conclusion is trivial. Now we assume that $D \neq 0$. Let $|D|=p_{1}^{l_{1}} p_{2}^{l_{2}} \ldots p_{r}^{l_{r}}$ be the standard factorization of $|D|$. Then

$$
r \log \left(\frac{1}{6 \log M} \log x\right) \leq \sum_{i=1}^{r} \log p_{i} \leq \log |D| \ll \log x .
$$

Thus

$$
r \ll \frac{\log x}{\log \log x}
$$

Hence

$$
\begin{aligned}
\sum_{\substack{a \in\left[c_{3} \sqrt{x}, c_{4} \sqrt{x}\right] \\
(a, D)>1}} 1 \leq \sum_{i=1}^{r} \sum_{\substack{a \in\left[c_{3} \sqrt{x}, c_{4} \sqrt{x}\right] \\
p_{i} \mid a}} 1 \leq \sum_{i=1}^{r}\left(\frac{\left(c_{4}-c_{3}\right) \sqrt{x}}{p_{i}}+1\right) \\
\leq \frac{\left(c_{4}-c_{3}\right) r \sqrt{x}}{\log x} 6 \log M+r \ll \frac{\sqrt{x}}{\log \log x} .
\end{aligned}
$$

This completes the proof of Lemma 2.

2. General lemmas. For an interval $I=(a, b]$, let

$$
\begin{gathered}
\left|I \sqrt{x} \cap A_{x}\right|=\alpha(I)|I| \sqrt{x}, \\
\left|I \sqrt{x} \cap A_{x} \cap(2 \mathbb{Z})\right|=\alpha^{(0)}(I)|I| \sqrt{x}, \\
\left|I \sqrt{x} \cap A_{x} \cap(2 \mathbb{Z}+1)\right|=\alpha^{(1)}(I)|I| \sqrt{x},
\end{gathered}
$$

where $|X|$ denotes the number of elements of $X$ or the length of an interval $X$, and $2 \mathbb{Z}$ and $2 \mathbb{Z}+1$ denote the sets of all even integers and all odd integers respectively. Let $\mathcal{I}=\left\{I_{1}, \ldots, I_{l}\right\}$ be a set of pairwise disjoint intervals with $I_{i}=\left(a_{i}, b_{i}\right]$ and $0<a_{0}<a_{1}<\ldots<a_{l}$. Let

$$
\alpha_{i}=\alpha\left(I_{i}\right), \quad \alpha_{i}^{(0)}=\alpha^{(0)}\left(I_{i}\right), \quad \alpha_{i}^{(1)}=\alpha^{(1)}\left(I_{i}\right), \quad M=4\left(1+\left[a_{l}^{2}\right]\right),
$$

where $\left[a_{l}^{2}\right]$ denotes the integral part of $a_{l}^{2}$. It is clear that $\alpha_{i}=\alpha_{i}^{(0)}+\alpha_{i}^{(1)}$.

Lemma 3. Let $r_{i j}\left(j=1, \ldots, k_{i} ; i=1, \ldots, l\right)$ be distinct integers with

$$
\left|r_{i j}-r_{u v}\right| \leq g\left(r_{i j}, r_{u v}\right) a_{i} a_{u},
$$


where $g(a, b)=1+\frac{1}{4}\left(1-(-1)^{a}\right)\left(1-(-1)^{b}\right)$. Let

$$
\begin{aligned}
& k_{i}^{(0)}=\left|\left\{r_{i j}: 2 \mid r_{i j}, j=1, \ldots, k_{i}\right\}\right|, \\
& k_{i}^{(1)}=k_{i}-k_{i}^{(0)}, \quad i=1, \ldots, l .
\end{aligned}
$$

Then

$$
\sum_{i=1}^{l}\left(k_{i}^{(0)} \alpha_{i}^{(0)}+k_{i}^{(1)} \alpha_{i}^{(1)}\right) \leq \frac{1}{2}+O\left((\log \log x)^{-1 / 2}\right),
$$

where $O$ depends only on $\mathcal{I}$.

Proof. Let $K=\sum_{i=1}^{l} k_{i}$. If $K=0$ or 1 , then by the definitions of $\alpha_{i}^{(0)}$ and $\alpha_{i}^{(1)}$ the assertion of Lemma 3 is true. In the following we assume that $K \geq 2$. Let $\delta$ be a small positive number which will be determined later, and let

$$
I_{i}(t)=\left(a_{i}+t \delta, a_{i}+(t+1) \delta\right] .
$$

For the (index) set

$$
\left\{t_{i j}: 0 \leq t_{i j} \leq\left|I_{i}\right| / \delta-1, t_{i j} \in \mathbb{Z}, j=1, \ldots, k_{i} ; i=1, \ldots, l\right\}
$$

we first show that

$$
\left|\bigcup_{i, j}\left(I_{i}\left(t_{i j}\right) \sqrt{x} \cap A_{x} \cap\left(2 \mathbb{Z}+r_{i j}\right)\right)\right| \leq \frac{1}{2} \delta \sqrt{x}+O\left(\frac{\sqrt{x}}{\log \log x}\right),
$$

where $O$ depends only on $\mathcal{I}$. To do this we consider the set

$$
\Delta(a)=\bigcup_{i, j}\left\{M ! l_{i j}+r_{i j}+2 a\right\},
$$

where $l_{i j}$ are integers which will be determined later such that

$$
\left(a_{i}+t_{i j} \delta\right) \sqrt{x} \leq M ! l_{i j}+r_{i j} \leq\left(a_{i}+t_{i j} \delta\right)\left(\sqrt{x}+x^{1 / 4}\right)
$$

hold for $j=1, \ldots, k_{i} ; i=1, \ldots, l$. For convenience we rewrite $\Delta(0)$ as

$$
\Delta(0)=\left\{M ! l_{1}+r_{1}, M ! l_{2}+r_{2}, \ldots, M ! l_{K}+r_{K}\right\} .
$$

Since $a_{i} a_{u}<M / 4(i, u=1, \ldots, l)$, by the conditions of Lemma 3 we have

$$
\left|r_{i}-r_{j}\right|<M / 2, \quad i, j=1, \ldots, K,
$$

whence $K \leq M / 2$. Now we take $l_{1}$ satisfying (1). Suppose that we have chosen $l_{1}, \ldots, l_{u}(u<K)$. By Lemma 1 for $x \geq x_{0}\left(M, a_{0} / M !\right)$ there exists a $l_{u+1}$ satisfying (1) such that each prime factor of

$$
\prod_{i=1}^{u}\left(\frac{M !}{r_{u+1}-r_{i}} l_{u+1}-\frac{M !}{r_{u+1}-r_{i}} l_{i}+1\right)
$$

exceeds

$$
\frac{1}{6 \log M} \log x .
$$


Thus by induction we have determined all $l_{u}(1 \leq u \leq K)$. Let

$$
D=\prod_{1 \leq v<u \leq K}\left(\frac{M !}{r_{u}-r_{v}} l_{u}-\frac{M !}{r_{u}-r_{v}} l_{v}+1\right) .
$$

Then each prime factor of $D$ exceeds

$$
\frac{1}{6 \log M} \log x
$$

and by (1),

$$
\begin{aligned}
|D| & \leq \prod_{1 \leq v<u \leq K}\left|M ! l_{u}+r_{u}-M ! l_{v}-r_{v}\right| \\
& \leq\left(2 b_{l} \sqrt{x}\right)^{K(K-1) / 2} \leq\left(2 b_{l}\right)^{M(M-1)} x^{M(M-1)} .
\end{aligned}
$$

By Lemma 2, the number of $a$ such that $(a, D)>1$ and $a \in\left(0, b_{l} \sqrt{x}\right.$ is $O(\sqrt{x} / \log \log x)$, where $O$ depends only on $\mathcal{I}$. Let

$$
B=\left\{a: a \in \bigcup_{i=1}^{l}\left(I_{i} \sqrt{x} \cap \mathbb{Z}\right),(a, D)=1\right\} .
$$

If $a \in(0, \delta \sqrt{x} / 2]$ and

$$
M ! l_{u}+r_{u}+2 a \in B, \quad M ! l_{v}+r_{v}+2 a \in B,
$$

then for $u \neq v$ we have

$$
\begin{aligned}
\left(M ! l_{u}+r_{u}+2 a, M ! l_{v}+r_{v}+2 a\right) & \\
& =\left(M ! l_{u}+r_{u}+2 a, M !\left(l_{v}-l_{u}\right)+r_{v}-r_{u}\right) \\
& =\left(M ! l_{u}+r_{u}+2 a, r_{v}-r_{u}\right) \leq g\left(r_{u}, r_{v}\right)^{-1}\left|r_{u}-r_{v}\right| .
\end{aligned}
$$

Thus for $a \in(0, \delta \sqrt{x} / 2]$ with

$$
\begin{aligned}
& M ! l_{i j}+r_{i j}+2 a \in B, \\
& M ! l_{u v}+r_{u v}+2 a \in B, \quad(i-u)^{2}+(j-v)^{2} \neq 0,
\end{aligned}
$$

by (1), (2) and the conditions of the lemma we have

$$
\begin{aligned}
\text { l.c.m. }\left\{M ! l_{i j}+r_{i j}+2 a, M ! l_{u v}\right. & \left.+r_{u v}+2 a\right\} \\
= & \frac{\left(M ! l_{i j}+r_{i j}+2 a\right)\left(M ! l_{u v}+r_{u v}+2 a\right)}{\left(M ! l_{i j}+r_{i j}+2 a, M ! l_{u v}+r_{u v}+2 a\right)} \\
& >\frac{\left(a_{i}+t_{i j} \delta\right)\left(a_{u}+t_{u v} \delta\right) x}{\left|r_{i j}-r_{u v}\right|} g\left(r_{i j}, r_{u v}\right) \\
& \geq \frac{\left(a_{i}+t_{i j} \delta\right)\left(a_{u}+t_{u v} \delta\right) x}{a_{i} a_{u}} \geq x .
\end{aligned}
$$


So $\left|\Delta(a) \cap B \cap A_{x}\right| \leq 1$. Since (see (1))

$$
\begin{aligned}
I_{i}\left(t_{i j}\right) \sqrt{x} \cap & \left(2 \mathbb{Z}+r_{i j}\right) \\
\subseteq & \left(\left(M ! l_{i j}+r_{i j}, M ! l_{i j}+r_{i j}+\delta \sqrt{x}\right]\right. \\
& \left.\cup\left(\left(a_{i}+t_{i j} \delta\right) \sqrt{x},\left(a_{i}+t_{i j} \delta\right)\left(\sqrt{x}+x^{1 / 4}\right)\right]\right) \cap\left(2 \mathbb{Z}+r_{i j}\right) \\
\subseteq & \left(\bigcup_{0<a \leq \delta \sqrt{x} / 2}\left\{M ! l_{i j}+r_{i j}+2 a\right\}\right) \\
& \cup\left(\left(\left(a_{i}+t_{i j} \delta\right) \sqrt{x},\left(a_{i}+t_{i j} \delta\right)\left(\sqrt{x}+x^{1 / 4}\right)\right] \cap \mathbb{Z}\right),
\end{aligned}
$$

we have

$$
\begin{aligned}
\bigcup_{i, j}\left(I_{i}\left(t_{i j}\right) \sqrt{x} \cap\right. & \left.\left(2 \mathbb{Z}+r_{i j}\right)\right) \\
\subseteq & \left(\bigcup_{0<a \leq \delta \sqrt{x} / 2} \bigcup_{i, j}\left\{M ! l_{i j}+r_{i j}+2 a\right\}\right) \\
& \cup\left(\bigcup_{i, j}\left(\left(\left(a_{i}+t_{i j} \delta\right) \sqrt{x},\left(a_{i}+t_{i j} \delta\right)\left(\sqrt{x}+x^{1 / 4}\right)\right] \cap \mathbb{Z}\right)\right) \\
\subseteq & \left(\bigcup_{0<a \leq \delta \sqrt{x} / 2} \Delta(a)\right) \\
& \cup\left(\bigcup_{i, j}\left(\left(\left(a_{i}+t_{i j} \delta\right) \sqrt{x},\left(a_{i}+t_{i j} \delta\right)\left(\sqrt{x}+x^{1 / 4}\right)\right] \cap \mathbb{Z}\right)\right) .
\end{aligned}
$$

Hence

$$
\begin{aligned}
& \left|\bigcup_{i, j}\left(I_{i}\left(t_{i j}\right) \sqrt{x} \cap A_{x} \cap B \cap\left(2 \mathbb{Z}+r_{i j}\right)\right)\right| \\
& \quad \leq \frac{1}{2} \delta \sqrt{x}+\sum_{i, j}\left(\left(a_{i}+t_{i j} \delta\right) x^{1 / 4}+1\right) \leq \frac{1}{2} \delta \sqrt{x}+\sum_{i, j}\left(\left(a_{i}+\left|I_{i}\right|\right) x^{1 / 4}+1\right) \\
& \quad \leq \frac{1}{2} \delta \sqrt{x}+\sum_{i, j}\left(b_{i} x^{1 / 4}+1\right) \leq \frac{1}{2} \delta \sqrt{x}+K \max _{i} b_{i} x^{1 / 4}+K \\
& \quad \leq \frac{1}{2} \delta \sqrt{x}+O\left(x^{1 / 4}\right),
\end{aligned}
$$

where $O$ depends only on $\mathcal{I}$ (note that $K \leq M$ ), whence

$$
\begin{aligned}
\mid \bigcup_{i, j}\left(I_{i}\left(t_{i j}\right) \sqrt{x}\right. & \left.\cap A_{x} \cap\left(2 \mathbb{Z}+r_{i j}\right)\right) \mid \\
\leq & \frac{1}{2} \delta \sqrt{x}+O\left(x^{1 / 4}\right)+O\left(\frac{\sqrt{x}}{\log \log x}\right) \leq \frac{1}{2} \delta \sqrt{x}+O\left(\frac{\sqrt{x}}{\log \log x}\right),
\end{aligned}
$$

where $O$ depends only on $\mathcal{I}$. Since $I_{1}, \ldots, I_{l}$ are pairwise disjoint, we have 


$$
\begin{aligned}
\left|\bigcup_{i \leq l-1} \bigcup_{j}\left(I_{i}\left(t_{i j}\right) \sqrt{x} \cap A_{x} \cap\left(2 \mathbb{Z}+r_{i j}\right)\right)\right| & +\left|\bigcup_{\substack{j=1 \\
2 \mid r_{l j}}}^{k_{l}}\left(I_{l}\left(t_{l j}\right) \sqrt{x} \cap A_{x} \cap(2 \mathbb{Z})\right)\right| \\
& +\left|\bigcup_{\substack{j=1 \\
k_{l} \\
2 \nmid r_{l j}}}^{\bigcup_{r_{l}}}\left(I_{l}\left(t_{l j}\right) \sqrt{x} \cap A_{x} \cap(2 \mathbb{Z}+1)\right)\right| \\
\leq & \frac{1}{2} \delta \sqrt{x}+O\left(\frac{\sqrt{x}}{\log \log x}\right) .
\end{aligned}
$$

Hence, if $k_{l}^{(1)} \geq 1$ and $u \geq 0$, then

$$
\begin{aligned}
\mid \bigcup_{i \leq l-1} \bigcup_{j}\left(I_{i}\left(t_{i j}\right) \sqrt{x} \cap\right. & \left.A_{x} \cap\left(2 \mathbb{Z}+r_{i j}\right)\right)|+| \bigcup_{\substack{j=1 \\
2 \mid r_{l j}}}^{k_{l}}\left(I_{l}\left(t_{l j}\right) \sqrt{x} \cap A_{x} \cap(2 \mathbb{Z})\right) \mid \\
& +\left|\bigcup_{\substack{r=0 \\
k_{l}^{(1)} u+r \leq\left|I_{l}\right| / \delta-1}}^{k_{l}^{(1)}-1}\left(I_{l}\left(k_{l}^{(1)} u+r\right) \sqrt{x} \cap A_{x} \cap(2 \mathbb{Z}+1)\right)\right| \\
\leq & \frac{1}{2} \delta \sqrt{x}+O\left(\frac{\sqrt{x}}{\log \log x}\right) .
\end{aligned}
$$

Thus

$$
\begin{aligned}
& \left(\left\lfloor\frac{\left|I_{l}\right|}{k_{l}^{(1)} \delta}\right]+1\right)\left|\bigcup_{i \leq l-1} \bigcup_{j}\left(I_{i}\left(t_{i j}\right) \sqrt{x} \cap A_{x} \cap\left(2 \mathbb{Z}+r_{i j}\right)\right)\right| \\
& \quad+\left(\left[\frac{\left|I_{l}\right|}{k_{l}^{(1)} \delta}\right]+1\right)\left|\bigcup_{\substack{j=1 \\
2 \mid r_{l j}}}^{k_{l}}\left(I_{l}\left(t_{l j}\right) \sqrt{x} \cap A_{x} \cap(2 \mathbb{Z})\right)\right| \\
& \quad+\sum_{0 \leq u \leq\left[\left|I_{l}\right| /\left(k_{l}^{(1)} \delta\right)\right]}\left|\bigcup_{\substack{r=0 \\
k_{l}^{(1)} u+r \leq\left|I_{l}\right| / \delta-1}}^{k_{l}^{(1)}-1}\left(I_{l}\left(k_{l}^{(1)} u+r\right) \sqrt{x} \cap A_{x} \cap(2 \mathbb{Z}+1)\right)\right| \\
& \leq \frac{1}{2} \delta \sqrt{x}\left(\left[\frac{\left|I_{l}\right|}{k_{l}^{(1)} \delta}\right]+1\right)+O\left(\left(\left[\frac{\left|I_{l}\right|}{k_{l}^{(1)} \delta}\right]+1\right) \frac{\sqrt{x}}{\log \log x}\right) .
\end{aligned}
$$

\section{Hence}

$$
\begin{aligned}
\frac{\left|I_{l}\right|}{k_{l}^{(1)} \delta} \mid \bigcup_{i \leq l-1} \bigcup_{j}\left(I_{i}\left(t_{i j}\right) \sqrt{x} \cap A_{x} \cap\right. & \left.\left(2 \mathbb{Z}+r_{i j}\right)\right) \mid \\
& +\frac{\left|I_{l}\right|}{k_{l}^{(1)} \delta}\left|\bigcup_{\substack{j=1 \\
2 \mid r_{l j}}}^{k_{l}}\left(I_{l}\left(t_{l j}\right) \sqrt{x} \cap A_{x} \cap(2 \mathbb{Z})\right)\right|
\end{aligned}
$$




$$
\begin{aligned}
& +\left|\bigcup_{0 \leq t \leq\left|I_{l}\right| / \delta-1}\left(I_{l}(t) \sqrt{x} \cap A_{x} \cap(2 \mathbb{Z}+1)\right)\right| \\
\leq & \frac{1}{2} \delta \sqrt{x}\left(\frac{\left|I_{l}\right|}{k_{l}^{(1)} \delta}+1\right)+O\left(\left(\frac{\left|I_{l}\right|}{k_{l}^{(1)} \delta}+1\right) \frac{\sqrt{x}}{\log \log x}\right) .
\end{aligned}
$$

So

$$
\begin{aligned}
&\left|\bigcup_{i \leq l-1} \bigcup_{j}\left(I_{i}\left(t_{i j}\right) \sqrt{x} \cap A_{x} \cap\left(2 \mathbb{Z}+r_{i j}\right)\right)\right| \\
&+\left|\bigcup_{j=1,2 \mid r_{l j}}^{k_{l}}\left(I_{l}\left(t_{l j}\right) \sqrt{x} \cap A_{x} \cap(2 \mathbb{Z})\right)\right| \\
&+\frac{k_{l}^{(1)} \delta}{\left|I_{l}\right|} \bigcup_{0 \leq t \leq\left|I_{l}\right| / \delta-1}\left(I_{l}(t) \sqrt{x} \cap A_{x} \cap(2 \mathbb{Z}+1)\right) \mid \\
& \leq \frac{1}{2} \delta \sqrt{x}+\frac{1}{2} \cdot \frac{k_{l}^{(1)} \delta^{2}}{\left|I_{l}\right|} \sqrt{x}+O\left(\left(1+\frac{k_{l}^{(1)} \delta}{\left|I_{l}\right|}\right) \frac{\sqrt{x}}{\log \log x}\right) \\
& \leq \frac{1}{2} \delta \sqrt{x}+\frac{1}{2} \cdot \frac{K \delta^{2}}{\left|I_{l}\right|} \sqrt{x}+O\left(\left(1+\frac{K \delta}{\left|I_{l}\right|}\right) \frac{\sqrt{x}}{\log \log x}\right) \\
& \leq \frac{1}{2} \delta \sqrt{x}+O\left(\delta^{2} \sqrt{x}+\frac{\sqrt{x}}{\log \log x}\right) .
\end{aligned}
$$

Noting that

$$
\begin{aligned}
\mid \bigcup_{0 \leq t \leq\left|I_{l}\right| / \delta-1}\left(I_{l}(t)\right. & \left.\sqrt{x} \cap A_{x} \cap(2 \mathbb{Z}+1)\right) \mid \\
= & \left|I_{l} \sqrt{x} \cap A_{x} \cap(2 \mathbb{Z}+1)\right|-\theta_{l}^{(1)} \delta \sqrt{x} \quad\left(0 \leq \theta_{l}^{(1)} \leq 1\right) \\
= & \alpha_{l}^{(1)}\left|I_{l}\right| \sqrt{x}-\theta_{l}^{(1)} \delta \sqrt{x},
\end{aligned}
$$

we have

(3)

$$
\begin{aligned}
& \left|\bigcup_{i \leq l-1} \bigcup_{j}\left(I_{i}\left(t_{i j}\right) \sqrt{x} \cap A_{x} \cap\left(2 \mathbb{Z}+r_{i j}\right)\right)\right| \\
& \quad+\left|\bigcup_{j=1,2 \mid r_{l j}}^{k_{l}}\left(I_{l}\left(t_{l j}\right) \sqrt{x} \cap A_{x} \cap(2 \mathbb{Z})\right)\right|+k_{l}^{(1)} \alpha_{l}^{(1)} \delta \sqrt{x} \\
& \leq \frac{1}{2} \delta \sqrt{x}+\theta_{l}^{(1)} \frac{k_{l}^{(1)} \delta^{2}}{\left|I_{l}\right|} \sqrt{x}+O\left(\delta^{2} \sqrt{x}+\frac{\sqrt{x}}{\log \log x}\right) \\
& \leq \frac{1}{2} \delta \sqrt{x}+O\left(\delta^{2} \sqrt{x}+\frac{\sqrt{x}}{\log \log x}\right) .
\end{aligned}
$$


It is clear that if $k_{l}^{(1)}=0,(3)$ also holds. Similarly, we have

$$
\begin{array}{r}
\left|\bigcup_{i \leq l-1} \bigcup_{j}\left(I_{i}\left(t_{i j}\right) \sqrt{x} \cap A_{x} \cap\left(2 \mathbb{Z}+r_{i j}\right)\right)\right|+k_{l}^{(0)} \alpha_{l}^{(0)} \delta \sqrt{x}+k_{l}^{(1)} \alpha_{l}^{(1)} \delta \sqrt{x} \\
\leq \frac{1}{2} \delta \sqrt{x}+O\left(\delta^{2} \sqrt{x}+\frac{\sqrt{x}}{\log \log x}\right) .
\end{array}
$$

Continuing this procedure we have

$$
\sum_{i=1}^{l}\left(k_{i}^{(0)} \alpha_{i}^{(0)}+k_{i}^{(1)} \alpha_{i}^{(1)}\right) \delta \sqrt{x} \leq \frac{1}{2} \delta \sqrt{x}+O\left(\delta^{2} \sqrt{x}+\frac{\sqrt{x}}{\log \log x}\right),
$$

where $O$ depends only on $\mathcal{I}$. Taking $\delta=(\log \log x)^{-1 / 2}$, we have

$$
\sum_{i=1}^{l}\left(k_{i}^{(0)} \alpha_{i}^{(0)}+k_{i}^{(1)} \alpha_{i}^{(1)}\right) \leq \frac{1}{2}+O\left((\log \log x)^{-1 / 2}\right) .
$$

This completes the proof of Lemma 3.

Corollary. Let the conditions be as in Lemma 3 and $m_{1}^{(0)}, \ldots, m_{l}^{(0)}$, $m_{1}^{(1)}, \ldots, m_{l}^{(1)}$ be nonnegative integers with

$$
\sum_{i=1}^{t} m_{i}^{(v)} \leq \sum_{i=1}^{t} k_{i}^{(v)}, \quad t=1, \ldots, l ; v=0,1 .
$$

Then

$$
\sum_{i=1}^{l}\left(m_{i}^{(0)} \alpha_{i}^{(0)}+m_{i}^{(1)} \alpha_{i}^{(1)}\right) \leq \frac{1}{2}+O\left((\log \log x)^{-1 / 2}\right),
$$

where $O$ depends only on $\mathcal{I}$.

Proof. Let $m_{i}=m_{i}^{(0)}+m_{i}^{(1)}$. By (4) we may rearrange $\left\{r_{i j}\right\}$ as

$$
\left\{w_{i j}: i=1, \ldots, l ; j=1, \ldots, m_{i}\right\} \cup A
$$

such that $w_{i j}=r_{u v}$ implies that $u \leq i$, and

$$
m_{i}^{(0)}=\left|\left\{w_{i j}: 2 \mid w_{i j}, j=1, \ldots, m_{i}\right\}\right| .
$$

Thus

$$
\left|w_{i j}-w_{i^{\prime} j^{\prime}}\right|=\left|r_{u v}-r_{u^{\prime} v^{\prime}}\right| \leq g\left(r_{u v}, r_{u^{\prime} v^{\prime}}\right) a_{u} a_{u^{\prime}} \leq g\left(w_{i j}, w_{i^{\prime} j^{\prime}}\right) a_{i} a_{i^{\prime}} .
$$

Then the Corollary follows from Lemma 3.

LEMma 4. Let $m, n_{1}, \ldots, n_{r}$ be nonnegative integers with $m \leq n_{1}+\ldots$ $\ldots+n_{r}$. Then there exist nonnegative integers $m_{1}, \ldots, m_{r}$ such that

$$
m=m_{1}+\ldots+m_{r} \quad \text { and } \quad m_{i} \leq n_{i}, \quad i=1, \ldots, r .
$$

The proof is clear. 
Lemma 5. Let the conditions be as in Lemma 3 . Let $\beta_{1}^{(v)}, \ldots, \beta_{l}^{(v)}(v=$ $0,1)$ be nonnegative real numbers with

$$
\sum_{i=1}^{t} \beta_{i}^{(v)} \leq \sum_{i=1}^{t} k_{i}^{(v)}, \quad t=1, \ldots, l ; v=0,1 .
$$

Then

$$
\sum_{i=1}^{l}\left(\beta_{i}^{(0)} \alpha_{i}^{(0)}+\beta_{i}^{(1)} \alpha_{i}^{(1)}\right) \leq \frac{1}{2}+O\left((\log \log x)^{-1 / 2}\right),
$$

where $O$ depends only on $\mathcal{I}$.

Proof. Let $n$ and $n_{i}^{(v)}(1 \leq i \leq l ; v=0,1)$ be nonnegative integers with

Then (5) implies that

$$
\frac{n_{i}^{(v)}}{n} \leq \beta_{i}^{(v)}<\frac{n_{i}^{(v)}+1}{n}, \quad i=1, \ldots, l ; v=0,1 .
$$

$$
\sum_{i=1}^{t} n_{i}^{(v)} \leq n \sum_{i=1}^{t} k_{i}^{(v)}, \quad t=1, \ldots, l ; v=0,1 .
$$

Now we use induction on $t$ to prove the following proposition $P(t)$ : There exist nonnegative integers $n_{i j}^{(v)}(1 \leq i \leq t ; 1 \leq j \leq n ; v=0,1)$ such that

$$
n_{i}^{(v)}=\sum_{j=1}^{n} n_{i j}^{(v)}, \quad v=0,1 ; i=1, \ldots, t
$$

and

$$
\sum_{i=1}^{s} n_{i j}^{(v)} \leq \sum_{i=1}^{s} k_{i}^{(v)}, \quad s=1, \ldots, t ; j=1, \ldots, n ; v=0,1 .
$$

By (6) and Lemma $4, P(1)$ is true. Suppose that $P(t)(1 \leq t<l)$ is true. Now by (6) and the induction hypothesis we have

$$
n_{t+1}^{(v)} \leq n \sum_{i=1}^{t+1} k_{i}^{(v)}-\sum_{i=1}^{t} n_{i}^{(v)} \leq \sum_{j=1}^{n}\left(\sum_{i=1}^{t+1} k_{i}^{(v)}-\sum_{i=1}^{t} n_{i j}^{(v)}\right)
$$

and

$$
\sum_{i=1}^{t+1} k_{i}^{(v)}-\sum_{i=1}^{t} n_{i j}^{(v)} \geq 0
$$

By Lemma 4 there exist nonnegative integers $n_{(t+1) j}^{(v)}(1 \leq j \leq n ; v=0,1)$ such that

$$
n_{(t+1) j}^{(v)} \leq \sum_{i=1}^{t+1} k_{i}^{(v)}-\sum_{i=1}^{t} n_{i j}^{(v)}, \quad j=1, \ldots, n,
$$


and

$$
n_{t+1}^{(v)}=\sum_{j=1}^{n} n_{(t+1) j}^{(v)}, \quad v=0,1 .
$$

So $P(t+1)$ is true. Hence $P(t)$ is true for all $t, 1 \leq t \leq l$. In particular, $P(l)$ is true, that is, there exist nonnegative integers $n_{i j}^{(v)}(1 \leq i \leq l ; 1 \leq j \leq n$; $v=0,1)$ such that

$$
n_{i}^{(v)}=\sum_{j=1}^{n} n_{i j}^{(v)}, \quad v=0,1 ; i=1, \ldots, l,
$$

and

$$
\sum_{i=1}^{t} n_{i j}^{(v)} \leq \sum_{i=1}^{t} k_{i}^{(v)}, \quad t=1, \ldots, l ; j=1, \ldots, n ; v=0,1 .
$$

By the Corollary of Lemma 3 we have

$$
\sum_{i=1}^{l}\left(n_{i j}^{(0)} \alpha_{i}^{(0)}+n_{i j}^{(1)} \alpha_{i}^{(1)}\right) \leq \frac{1}{2}+O\left((\log \log x)^{-1 / 2}\right), \quad j=1, \ldots, n .
$$

Hence

$$
\sum_{i=1}^{l}\left(n_{i}^{(0)} \alpha_{i}^{(0)}+n_{i}^{(1)} \alpha_{i}^{(1)}\right) \leq \frac{1}{2} n+O\left(n(\log \log x)^{-1 / 2}\right)
$$

that is,

$$
\sum_{i=1}^{l}\left(\frac{n_{i}^{(0)}}{n} \alpha_{i}^{(0)}+\frac{n_{i}^{(1)}}{n} \alpha_{i}^{(1)}\right) \leq \frac{1}{2}+O\left((\log \log x)^{-1 / 2}\right),
$$

where $O$ depends only on $\mathcal{I}$. Letting $n \rightarrow \infty$ we obtain the statement of Lemma 5.

Lemma 6. Let

$$
\sum_{i=1}^{l}\left(k_{i j}^{(0)} \alpha_{i}^{(0)}+k_{i j}^{(1)} \alpha_{i}^{(1)}\right) \leq \frac{1}{2}+O\left((\log \log x)^{-1 / 2}\right)
$$

$(j=1, \ldots, r)$ be $r$ relations obtained by using Lemma 3 (not necessarily from the same $\left.\left\{r_{i j}\right\}\right)$. Let $\beta_{1}^{(v)}, \ldots, \beta_{l}^{(v)}, \delta_{1}, \ldots, \delta_{r}(v=0,1)$ be nonnegative real numbers with

$$
\sum_{i=1}^{t} \beta_{i}^{(v)} \leq \sum_{i=1}^{t} \sum_{j=1}^{r} \delta_{j} k_{i j}^{(v)}, \quad t=1, \ldots, l ; v=0,1 .
$$

Then

$$
\sum_{i=1}^{l}\left(\beta_{i}^{(0)} \alpha_{i}^{(0)}+\beta_{i}^{(1)} \alpha_{i}^{(1)}\right) \leq \frac{1}{2} \sum_{j=1}^{r} \delta_{j}+O\left(\sum_{j=1}^{r} \delta_{j}(\log \log x)^{-1 / 2}\right),
$$

where $O$ depends only on $\mathcal{I}$. 
Proof. As in Lemma 4 , if $u$ and $v_{j}(1 \leq j \leq r)$ are nonnegative real numbers with $u \leq \sum_{j=1}^{r} \delta_{j} v_{j}$, then there exist nonnegative real numbers $u_{1}, \ldots, u_{r}$ such that

$$
u=\sum_{j=1}^{r} \delta_{j} u_{j}, \quad u_{j} \leq v_{j}, \quad j=1, \ldots, r .
$$

Using this fact and (7) we infer, as in the proof of Lemma 5, that there exist nonnegative real numbers $\beta_{i j}^{(v)}(i=1, \ldots, l ; j=1, \ldots, r ; v=0,1)$ such that

$$
\beta_{i}^{(v)}=\sum_{j=1}^{r} \delta_{j} \beta_{i j}^{(v)}, \quad i=1, \ldots, l ; v=0,1,
$$

and

$$
\sum_{i=1}^{t} \beta_{i j}^{(v)} \leq \sum_{i=1}^{t} k_{i j}^{(v)}, \quad t=1, \ldots, l ; j=1, \ldots, r ; v=0,1 .
$$

By Lemma 5 we have

$$
\sum_{i=1}^{l}\left(\beta_{i j}^{(0)} \alpha_{i}^{(0)}+\beta_{i j}^{(1)} \alpha_{i}^{(1)}\right) \leq \frac{1}{2}+O\left((\log \log x)^{-1 / 2}\right) .
$$

Hence

$\sum_{i=1}^{l}\left(\sum_{j=1}^{r} \delta_{j} \beta_{i j}^{(0)} \alpha_{i}^{(0)}+\sum_{j=1}^{r} \delta_{j} \beta_{i j}^{(1)} \alpha_{i}^{(1)}\right) \leq \frac{1}{2} \sum_{j=1}^{r} \delta_{j}+O\left(\sum_{j=1}^{r} \delta_{j}(\log \log x)^{-1 / 2}\right)$.

That is,

$$
\sum_{i=1}^{l}\left(\beta_{i}^{(0)} \alpha_{i}^{(0)}+\beta_{i}^{(1)} \alpha_{i}^{(1)}\right) \leq \frac{1}{2} \sum_{j=1}^{r} \delta_{j}+O\left(\sum_{j=1}^{r} \delta_{j}(\log \log x)^{-1 / 2}\right),
$$

where $O$ depends only on $\mathcal{I}$. This completes the proof of Lemma 6 .

Lemma 7. Let $r_{i j}\left(j=1, \ldots, k_{i} ; i=1, \ldots, l\right)$ be distinct integers with

$$
\left|r_{i j}-r_{u v}\right| \leq a_{i} a_{u}
$$

Then

$$
\sum_{i=1}^{l} k_{i} \alpha_{i} \leq 1+O\left((\log \log x)^{-1 / 2}\right),
$$

where $O$ depends only on $\mathcal{I}$.

Proof. By Lemma 3 we have

$$
\sum_{i=1}^{l}\left(k_{i}^{(0)} \alpha_{i}^{(0)}+k_{i}^{(1)} \alpha_{i}^{(1)}\right) \leq \frac{1}{2}+O\left((\log \log x)^{-1 / 2}\right) .
$$


Let

$$
\begin{array}{cl} 
& w_{i j}=r_{i j}+1 \\
n_{i}=k_{i}, \quad j=1, \ldots, n_{i} ; i=1, \ldots, l .
\end{array}
$$

Then

$$
\left|w_{i j}-w_{u v}\right| \leq a_{i} a_{u}, \quad n_{i}^{(0)}=k_{i}^{(1)}, \quad n_{i}^{(1)}=k_{i}^{(0)} .
$$

By Lemma 3 we have

$$
\sum_{i=1}^{l}\left(n_{i}^{(0)} \alpha_{i}^{(0)}+n_{i}^{(1)} \alpha_{i}^{(1)}\right) \leq \frac{1}{2}+O\left((\log \log x)^{-1 / 2}\right) .
$$

That is,

$$
\sum_{i=1}^{l}\left(k_{i}^{(1)} \alpha_{i}^{(0)}+k_{i}^{(0)} \alpha_{i}^{(1)}\right) \leq \frac{1}{2}+O\left((\log \log x)^{-1 / 2}\right) .
$$

By (8), (9), $k_{i}^{(0)}+k_{i}^{(1)}=k_{i}$ and $\alpha_{i}^{(0)}+\alpha_{i}^{(1)}=\alpha_{i}$, we have

$$
\sum_{i=1}^{l} k_{i} \alpha_{i} \leq 1+O\left((\log \log x)^{-1 / 2}\right) .
$$

This completes the proof of Lemma 7.

LEMMA 8. Let

$$
\sum_{i=1}^{l} k_{i j} \alpha_{i} \leq 1+O\left((\log \log x)^{-1 / 2}\right), \quad j=1, \ldots, r,
$$

be $r$ relations obtained by using Lemma 7 (not necessarily from the same $\left.\left\{r_{i j}\right\}\right)$. Let $\beta_{1}, \ldots, \beta_{l}, \delta_{1}, \ldots, \delta_{r}$ be nonnegative real numbers with

$$
\sum_{i=1}^{t} \beta_{i} \leq \sum_{i=1}^{t} \sum_{j=1}^{r} \delta_{j} k_{i j}, \quad t=1, \ldots, l .
$$

Then

$$
\sum_{i=1}^{l} \beta_{i} \alpha_{i} \leq \sum_{j=1}^{r} \delta_{j}+O\left(\sum_{j=1}^{r} \delta_{j}(\log \log x)^{-1 / 2}\right),
$$

where $O$ depends only on $\mathcal{I}$.

Proof. By (10) and $k_{i j}=k_{i j}^{(0)}+k_{i j}^{(1)}$ we have

$$
\sum_{i=1}^{t} \beta_{i} \leq \sum_{i=1}^{t} \sum_{j=1}^{r} \delta_{j} k_{i j}^{(0)}+\sum_{i=1}^{t} \sum_{j=1}^{r} \delta_{j} k_{i j}^{(1)}, \quad t=1, \ldots, l .
$$


By the argument used in the proof of Lemma 5 there exist nonnegative real numbers $\beta_{i}^{(v)}(1 \leq i \leq l ; v=0,1)$ such that

$$
\sum_{i=1}^{t} \beta_{i}^{(v)} \leq \sum_{i=1}^{t} \sum_{j=1}^{r} \delta_{j} k_{i j}^{(v)}, \quad t=1, \ldots, l ; v=0,1 .
$$

By the argument used in the proof of Lemma 7 we have for $j=1, \ldots, r$,

$$
\begin{aligned}
& \sum_{i=1}^{l}\left(k_{i}^{(0)} \alpha_{i}^{(0)}+k_{i}^{(1)} \alpha_{i}^{(1)}\right) \leq \frac{1}{2}+O\left((\log \log x)^{-1 / 2}\right), \\
& \sum_{i=1}^{l}\left(k_{i}^{(1)} \alpha_{i}^{(0)}+k_{i}^{(0)} \alpha_{i}^{(1)}\right) \leq \frac{1}{2}+O\left((\log \log x)^{-1 / 2}\right) .
\end{aligned}
$$

By Lemma 6 we have

$$
\begin{aligned}
& \sum_{i=1}^{l}\left(\beta_{i}^{(0)} \alpha_{i}^{(0)}+\beta_{i}^{(1)} \alpha_{i}^{(1)}\right) \leq \frac{1}{2} \sum_{j=1}^{r} \delta_{j}+O\left(\sum_{j=1}^{r} \delta_{j}(\log \log x)^{-1 / 2}\right), \\
& \sum_{i=1}^{l}\left(\beta_{i}^{(1)} \alpha_{i}^{(0)}+\beta_{i}^{(0)} \alpha_{i}^{(1)}\right) \leq \frac{1}{2} \sum_{j=1}^{r} \delta_{j}+O\left(\sum_{j=1}^{r} \delta_{j}(\log \log x)^{-1 / 2}\right) .
\end{aligned}
$$

Since $\alpha_{i}=\alpha_{i}^{(0)}+\alpha_{i}^{(1)}$ and $\beta_{i}=\beta_{i}^{(0)}+\beta_{i}^{(1)}$, we have

$$
\sum_{i=1}^{l} \beta_{i} \alpha_{i} \leq \sum_{j=1}^{r} \delta_{j}+O\left(\sum_{j=1}^{r} \delta_{j}(\log \log x)^{-1 / 2}\right) .
$$

This completes the proof of Lemma 8.

3. The asymptotic formula for $\left|A_{x}\right|$. Let $L$ and $S$ be suitable large integers and

$$
q=2^{1 /(2 L)}, \quad I_{i}=\left(q^{i}, q^{i+1}\right], \quad T=2 L S-1 .
$$

For positive real numbers $\alpha, \beta$, let

$$
\begin{aligned}
B(\alpha, \beta)= & \{a: a \in \mathbb{Z}, 1 \leq a \leq \alpha \beta\} \\
& \cup\left\{a: a \in \mathbb{Z},-\min \left\{\alpha \beta, \alpha^{-1} \beta-1\right\} \leq a \leq 0\right\}, \\
A_{i j}= & \begin{cases}B\left(q^{j}, q^{i}\right) & \text { if } i \geq j, \\
\emptyset & \text { if } i<j .\end{cases}
\end{aligned}
$$

In the following we make the convention that $\sum_{a \in \emptyset} h(a)=0$ for any function $h(t)$.

Lemma 9. Let $0<\alpha \leq \min \{\beta, \gamma\}$. If $a \in B(\alpha, \beta)$ and $b \in B(\alpha, \gamma)$, then $|a-b| \leq \beta \gamma$.

Pro of. If $a b \geq 0$, then $|a-b| \leq \max \{|a|,|b|\} \leq \max \{\alpha \beta, \alpha \gamma\} \leq \beta \gamma$. 
Now we assume that $a b<0$. Without loss of generality, we may assume that $a>0$ and $b<0$. In this case we have $\alpha \beta \geq 1$ and $\alpha \gamma \geq 1$. Thus

$$
\begin{aligned}
|a-b| & =a-b \leq \alpha \beta+\min \left\{\alpha \gamma, \alpha^{-1} \gamma-1\right\} \\
& \leq \alpha \beta+\alpha^{-1} \gamma-1=\beta \gamma+\left(\beta-\alpha^{-1}\right)(\alpha-\gamma) \leq \beta \gamma .
\end{aligned}
$$

This completes the proof of Lemma 9.

To use Lemma 8, let

$$
\begin{aligned}
\alpha & =(10-7 \sqrt{2}) / 32, \\
k_{i j} & =\left|A_{i j} \backslash A_{(i-1) j}\right|,-T \leq i \leq T,-T \leq j \leq L-1, \\
k_{i L} & =0(-T \leq i \leq T, i \neq 0), k_{0 L}=1, \\
\beta_{i} & =q^{i}(q-1),-T \leq i \leq L-1, \\
\beta_{i} & =(1+\alpha) q^{i}(q-1), L \leq i \leq T, \\
\delta_{j} & =q^{j}(q-1),-T \leq j \leq-1, \\
\delta_{j} & =\frac{1}{2}(q-1)\left(q^{j}-q^{-j-1}\right), 0 \leq j \leq L-1, \\
\delta_{L} & =1-q^{-1} .
\end{aligned}
$$

Lemma 10. For $-T \leq j \leq L$, we have

$$
\sum_{-T \leq i \leq T} k_{i j} \alpha_{i} \leq 1+O\left((\log \log x)^{-1 / 2}\right),
$$

where $O$ depends only on $L$ and $S$.

Proof. The inequality

$$
\sum_{i=-T}^{T} k_{i L} \alpha_{i} \leq 1+O\left((\log \log x)^{-1 / 2}\right)
$$

can be deduced from Lemma 7 by taking

$$
\left\{r_{i j}: j=1, \ldots, k_{i L} ; i=-T, \ldots, T\right\}=\left\{r_{01}=1\right\} .
$$

Now we assume that $-T \leq j \leq L-1$. If $i<j$, then

$$
\left|A_{i j} \backslash A_{(i-1) j}\right|=\emptyset \text {. }
$$

If $j \leq i \leq i^{\prime} \leq T$ and

$$
a \in A_{i j} \backslash A_{(i-1) j}, \quad b \in A_{i^{\prime} j} \backslash A_{\left(i^{\prime}-1\right) j},
$$

then by Lemma 9 we have $|a-b| \leq q^{i+i^{\prime}}$. Then Lemma 10 follows from Lemma 7.

Lemma 11. There exists a $L_{0}$ such that if $L \geq L_{0}$, then

$$
\sum_{i=-T}^{t} \beta_{i} \leq \sum_{i=-T}^{t} \sum_{j=-T}^{L} \delta_{j} k_{i j}, \quad t=-T,-T+1, \ldots, T .
$$


Pr o of. For convenience let

$$
f(t)=\sum_{i=-T}^{t} \sum_{j=-T}^{L} \delta_{j} k_{i j} .
$$

That is,

$$
\begin{aligned}
f(t)= & \sum_{-T \leq i \leq t}\left(\sum_{-T \leq j \leq-1}\left|A_{i j} \backslash A_{(i-1) j}\right| q^{j}(q-1)\right. \\
& \left.+\frac{1}{2} \sum_{0 \leq j \leq L-1}\left|A_{i j} \backslash A_{(i-1) j}\right|(q-1)\left(q^{j}-q^{-j-1}\right)\right)+\varepsilon_{t}\left(1-q^{-1}\right),
\end{aligned}
$$

where $\varepsilon_{t}=0$ if $t \leq-1$, and $\varepsilon_{t}=1$ if $t \geq 0$. Since

$$
A_{i j} \supseteq A_{(i-1) j}, \quad A_{(-T-1) j}=\emptyset, \quad j \geq-T,
$$

we have

$$
\sum_{i=-T}^{t}\left|A_{i j} \backslash A_{(i-1) j}\right|=\left|A_{t j}\right|, \quad j \geq-T .
$$

Hence

$$
\begin{aligned}
f(t)= & \sum_{-T \leq j \leq-1} \sum_{-T \leq i \leq t}\left|A_{i j} \backslash A_{(i-1) j}\right| q^{j}(q-1) \\
& +\frac{1}{2} \sum_{0 \leq j \leq L-1} \sum_{-T \leq i \leq t}\left|A_{i j} \backslash A_{(i-1) j}\right|(q-1)\left(q^{j}-q^{-j-1}\right) \\
& +\varepsilon_{t}\left(1-q^{-1}\right) \\
= & \sum_{-T \leq j \leq-1}\left|A_{t j}\right| q^{j}(q-1) \\
& +\frac{1}{2} \sum_{0 \leq j \leq L-1}\left|A_{t j}\right|(q-1)\left(q^{j}-q^{-j-1}\right)+\varepsilon_{t}\left(1-q^{-1}\right) .
\end{aligned}
$$

Case 1: $-T \leq t \leq-1$. Then by (11) we have

$$
f(t) \geq \sum_{-T \leq j \leq t}\left|A_{t j}\right| q^{j}(q-1) \geq \sum_{-T \leq j \leq t} q^{j}(q-1) \geq \sum_{-T \leq i \leq t} \beta_{i} .
$$

CASE 2: $0 \leq t \leq L-1$. Then by (11) we have

$$
\begin{aligned}
f(t) \geq & \sum_{-T \leq j \leq-t-1}\left|A_{t j}\right| q^{j}(q-1)+\sum_{-t \leq j \leq-1}\left|A_{t j}\right| q^{j}(q-1) \\
& +\frac{1}{2} \sum_{0 \leq j \leq t}\left|A_{t j}\right|(q-1)\left(q^{j}-q^{-j-1}\right)+1-q^{-1} \\
\geq & \sum_{-T \leq j \leq-t-1} q^{j}(q-1)+2 \sum_{-t \leq j \leq-1} q^{j}(q-1)
\end{aligned}
$$




$$
\begin{aligned}
& +\sum_{0 \leq j \leq t}(q-1)\left(q^{j}-q^{-j-1}\right)+1-q^{-1} \\
\geq & q^{t+1}-q^{-T}+\left(1-q^{-1}\right)\left(1-q^{-t}\right) \\
\geq & q^{t+1}-q^{-T} \geq \sum_{-T \leq i \leq t} \beta_{i} .
\end{aligned}
$$

CASE 3: $L \leq t \leq 2 L-1$. Then by (11) we have

$$
\begin{aligned}
f(t) \geq & \sum_{-T \leq j \leq-t-1}\left|A_{t j}\right| q^{j}(q-1)+\sum_{-t \leq j \leq t-2 L}\left|A_{t j}\right| q^{j}(q-1) \\
& +\sum_{t-2 L<j \leq-1}\left|A_{t j}\right| q^{j}(q-1)+\frac{1}{2} \sum_{0 \leq j<2 L-t}\left|A_{t j}\right|(q-1)\left(q^{j}-q^{-j-1}\right) \\
& +\frac{1}{2} \sum_{2 L-t \leq j \leq L-1}\left|A_{t j}\right|(q-1)\left(q^{j}-q^{-j-1}\right)+1-q^{-1} \\
\geq & \sum_{-T \leq j \leq-t-1} q^{j}(q-1)+3 \sum_{-t \leq j \leq t-2 L} q^{j}(q-1)+2 \sum_{t-2 L<j \leq-1} q^{j}(q-1) \\
& +\sum_{0 \leq j<2 L-t}(q-1)\left(q^{j}-q^{-j-1}\right)+\frac{3}{2} \sum_{2 L-t \leq j \leq L-1}(q-1)\left(q^{j}-q^{-j-1}\right) \\
& +1-q^{-1} \geq \\
\geq & q^{t+1}-q^{-T}+\alpha\left(q^{t+1}-q^{L}\right)+\frac{9}{4} \sqrt{2}+\sqrt{2} \alpha+1-q^{-1} \\
& +q^{t}\left(-\frac{1}{2} q-\frac{1}{4}-\alpha q\right)-3 q^{-t} \\
\geq & q^{t+1}-q^{-T}+\alpha\left(q^{t+1}-q^{L}\right)+\frac{9}{4} \sqrt{2}+\sqrt{2} \alpha+1-q^{-1} \\
& +\min \left\{q^{L}\left(-\frac{1}{2} q-\frac{1}{4}-\alpha q\right)-3 q^{-L}, q^{2 L-1}\left(-\frac{1}{2} q-\frac{1}{4}-\alpha q\right)\right. \\
\geq & q^{t+1}-q^{-T}+\alpha\left(q^{t+1}-q^{L}\right) \\
\geq & \sum_{T \leq i \leq t} \beta_{i} .
\end{aligned}
$$

The last inequality but one holds for all sufficiently large $L$ (note that $q=$ $\left.2^{1 /(2 L)}\right)$.

CASE $4: T \geq t \geq 2 L$. Then by (11) we have

(12) $f(t) \geq \sum_{-T \leq j \leq-1}\left|A_{t j}\right| q^{j}(q-1)+\frac{1}{2} \sum_{0 \leq j \leq L-1}\left|A_{t j}\right|(q-1)\left(q^{j}-q^{-j-1}\right)$ 


$$
\begin{aligned}
= & \sum_{-T \leq j \leq-1} q^{j}(q-1)\left(\sum_{1 \leq a \leq q^{j+t}} 1+\sum_{-\min \left\{q^{j+t}, q^{t-j}-1\right\} \leq a \leq 0} 1\right) \\
& +\frac{1}{2} \sum_{0 \leq j \leq L-1}(q-1)\left(q^{j}-q^{-j-1}\right)\left(\sum_{1 \leq a \leq q^{j+t}} 1+\sum_{1-q^{t-j} \leq a \leq 0} 1\right) .
\end{aligned}
$$

Now we estimate each part in (12):

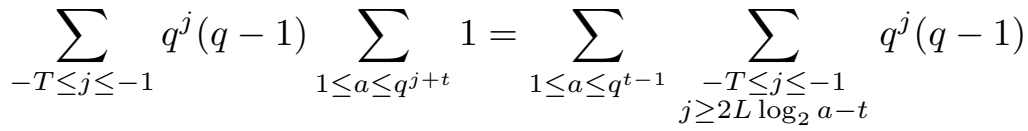

$$
\begin{aligned}
& \geq \sum_{1 \leq a \leq q^{t-1}}\left(1-q^{2 L \log _{2} a-t+1}\right) \\
& \geq \sum_{1 \leq a \leq q^{t}}\left(1-q^{2 L \log _{2} a-t+1}\right) \\
& \geq \sum_{1 \leq a \leq q^{t}}\left(1-a q^{-t+1}\right) \\
& \geq\left[q^{t}\right]-\frac{1}{2} q^{-t+1}\left[q^{t}\right]\left(\left[q^{t}\right]+1\right) ; \\
& \sum_{-T \leq j \leq-1} q^{j}(q-1) \sum_{-\min \left\{q^{t+j}, q^{t-j}-1\right\} \leq a \leq 0} 1 \\
& =\sum_{-T \leq j \leq-1} q^{j}(q-1) \sum_{1 \leq a \leq \min \left\{q^{t+j}+1, q^{t-j}\right\}} 1 \\
& \geq \sum_{1 \leq a \leq 1 / 2+q^{t}} \sum_{\substack{-T \leq j \leq-1 \\
\min \left\{q^{t+j}+1, q^{t-j}\right\} \geq a}} q^{j}(q-1) \\
& =: \sum_{2 \leq a \leq q^{t}} \sum_{\substack{-T \leq j \leq-1 \\
\min \left\{q^{t+j}+1, q^{t-j}\right\} \geq a}} q^{j}(q-1)+\delta(t)+1-q^{-T} \\
& =\sum_{2 \leq a \leq q^{t}} \sum_{2 L \log _{2}(a-1)-t \leq j \leq-1} q^{j}(q-1)+\delta(t)+1-q^{-T} \\
& \geq \sum_{2 \leq a \leq q^{t}}\left(1-q^{2 L \log _{2}(a-1)-t+1}\right)+\delta(t)+1-q^{-T} \\
& \geq \sum_{2 \leq a \leq q^{t}}\left(1-(a-1) q^{-t+1}\right)+\delta(t)+1-q^{-T} \\
& \geq\left[q^{t}\right]-\frac{1}{2} q^{-t+1}\left[q^{t}\right]\left(\left[q^{t}\right]-1\right)-q^{-T}+\delta(t) ;
\end{aligned}
$$




$$
\begin{aligned}
\sum_{0 \leq j \leq L-1}(q-1) & \left(q^{j}-q^{-j-1}\right)\left(\sum_{1 \leq a \leq q^{j+t}} 1+\sum_{1-q^{t-j} \leq a \leq 0} 1\right) \\
& \geq \sum_{0 \leq j \leq L-1}(q-1)\left(q^{j}-q^{-j-1}\right)\left(q^{j+t}+q^{t-j}-2\right) \\
& \geq \frac{q^{t}}{1+q}\left(1-\frac{1}{2} q\right)+L(q-1) q^{t}\left(1-q^{-1}\right)+4-3 \sqrt{2} \\
& \geq \frac{q^{t}}{1+q}\left(1-\frac{1}{2} q\right)+4-3 \sqrt{2} .
\end{aligned}
$$

Thus by these estimates and (12) we have

$$
\begin{aligned}
f(t) \geq & {\left[q^{t}\right]-\frac{1}{2} q^{-t+1}\left[q^{t}\right]\left(\left[q^{t}\right]+1\right) } \\
& +\left[q^{t}\right]-\frac{1}{2} q^{-t+1}\left[q^{t}\right]\left(\left[q^{t}\right]-1\right)-q^{-T}+\delta(t) \\
& +\frac{1}{2}\left(1-\frac{1}{2} q\right) \frac{q^{t}}{1+q}+2-\frac{3}{2} \sqrt{2} \\
\geq & 2\left[q^{t}\right]-q^{-t+1}\left[q^{t}\right]^{2}-q^{-T}+\delta(t) \\
& +\frac{1}{2}\left(1-\frac{1}{2} q\right) \frac{q^{t}}{1+q}+2-\frac{3}{2} \sqrt{2} .
\end{aligned}
$$

If $q^{t}-1 / 2 \leq\left[q^{t}\right] \leq q^{t}$ and $q^{t} \geq 2$ (i.e. $t \geq 2 L$ ), then by (13) and $\delta(t) \geq 0$ we have

$$
\begin{aligned}
f(t) \geq & \min \left\{2 q^{t}-q^{-t+1} q^{2 t}, 2\left(q^{t}-\frac{1}{2}\right)-q^{-t+1}\left(q^{t}-\frac{1}{2}\right)^{2}\right\} \\
& -q^{-T}+\frac{1}{2}\left(1-\frac{1}{2} q\right) \frac{q^{t}}{1+q}+2-\frac{3}{2} \sqrt{2} \\
\geq & q^{t+1}-q^{-T}+\alpha\left(q^{t+1}-q^{L}\right)+q^{t}\left(2-2 q+\frac{1}{2}\left(1-\frac{1}{2} q\right) \frac{1}{1+q}-\alpha q\right) \\
& +2-\frac{3}{2} \sqrt{2}+\sqrt{2} \alpha-\frac{1}{4 q^{t}} q \\
\geq & q^{t+1}-q^{-T}+\alpha\left(q^{t+1}-q^{L}\right) \\
& +2\left(2-2 q+\frac{1}{2}\left(1-\frac{1}{2} q\right) \frac{1}{1+q}-\alpha q\right)+2-\frac{3}{2} \sqrt{2}+\sqrt{2} \alpha-\frac{1}{8} q \\
\geq & q^{t+1}-q^{-T}+\alpha\left(q^{t+1}-q^{L}\right) \\
\geq & \sum_{-T \leq i \leq t} \beta_{i} .
\end{aligned}
$$

The last three inequalities hold for all sufficiently large $L$. If $q^{t}-1<\left[q^{t}\right]<$ 
$q^{t}-1 / 2$ and $q^{t} \geq 2$ (i.e. $t \geq 2 L$ ), then

$$
\begin{aligned}
\delta(t) & =\sum_{q^{t}<a \leq 1 / 2+q^{t}} \sum_{\substack{-T \leq j \leq-1 \\
\min \left\{q^{t+j}+1, q^{t-j}\right\} \geq a}} q^{j}(q-1) \\
& \geq \sum_{\min \left\{q^{t+j}+1, q^{t-j}\right\} \geq\left[q^{t}\right]+1} q^{j}(q-1) \\
& \geq \sum_{2 L \log _{2}\left[q^{t}\right]-t \leq j \leq t-2 L \log _{2}\left(\left[q^{t}\right]+1\right)} q^{j}(q-1) \\
& \geq q^{t-2 L \log _{2}\left(\left[q^{t}\right]+1\right)}-q^{2 L \log _{2}\left[q^{t}\right]-t+1} \geq \frac{1}{\left[q^{t}\right]+1} q^{t}-\left[q^{t}\right] q^{-t+1} .
\end{aligned}
$$

In this subcase, by (13) we have

$$
\begin{aligned}
f(t) \geq & 2\left[q^{t}\right]-q^{-t+1}\left[q^{t}\right]^{2}+\frac{1}{\left[q^{t}\right]+1} q^{t}-\left[q^{t}\right] q^{-t+1} \\
& -q^{-T}+\frac{1}{2}\left(1-\frac{1}{2} q\right) \frac{q^{t}}{1+q}+2-\frac{3}{2} \sqrt{2} \\
\geq & \min \left\{2\left(q^{t}-1\right)-q^{-t+1}\left(q^{t}-1\right)^{2}+\frac{1}{q^{t}-1+1} q^{t}-\left(q^{t}-1\right) q^{-t+1},\right. \\
& \left.2\left(q^{t}-\frac{1}{2}\right)-q^{-t+1}\left(q^{t}-\frac{1}{2}\right)^{2}+\frac{1}{q^{t}-1 / 2+1} q^{t}-\left(q^{t}-\frac{1}{2}\right) q^{-t+1}\right\} \\
& -q^{-T}+\frac{1}{2}\left(1-\frac{1}{2} q\right) \frac{q^{t}}{1+q}+2-\frac{3}{2} \sqrt{2} \\
\geq & q^{t+1}-q^{-T}+\alpha\left(q^{t+1}-q^{L}\right) \\
& +q^{t}\left(\frac{1}{2}\left(1-\frac{1}{2} q\right) \frac{1}{1+q}+2-2 q-\alpha q\right)-\frac{1}{4 q^{t-1}}+2-\frac{3}{2} \sqrt{2}+\alpha \sqrt{2} \\
\geq & q^{t+1}-q^{-T}+\alpha\left(q^{t+1}-q^{L}\right) \\
& +2\left(\frac{1}{2}\left(1-\frac{1}{2} q\right) \frac{1}{1+q}+2-2 q-\alpha q\right)-\frac{1}{8} q+2-\frac{3}{2} \sqrt{2}+\alpha \sqrt{2} \\
\geq & q^{t+1}-q^{-T}+\alpha\left(q^{t+1}-q^{L}\right) \\
\geq & \sum_{-} \beta_{i} .
\end{aligned}
$$

The last three inequalities hold for all sufficiently large $L$. This completes the proof of Lemma 11.

Lemma 12. Let $D$ be a positive integer and $x \geq 2 D$. Then

$$
\left|(\sqrt{D x}, x] \cap A_{x}\right|=O\left(\frac{1}{\sqrt{D}} \sqrt{x}\right),
$$

where the $O$-constant is absolute. 
Proof. For any integer $k \geq 1$, since for $a \geq \sqrt{k x}$,

$$
\left|\{a, a+1, \ldots, a+k-1\} \cap A_{x}\right| \leq 1,
$$

we have

$$
\left|[\sqrt{k x}, 2 \sqrt{k x}] \cap A_{x}\right| \leq \frac{1}{k}(2 \sqrt{k}-\sqrt{k}) \sqrt{x}+1=\frac{1}{\sqrt{k}} \sqrt{x}+1 .
$$

Thus

$$
\begin{aligned}
\left|(\sqrt{D x}, x] \cap A_{x}\right| & =\sum_{1 \leq i \leq \frac{1}{2} \log _{2}(x / D)+1}\left|\left(2^{i-1} \sqrt{D x}, 2^{i} \sqrt{D x}\right] \cap A_{x}\right| \\
& \leq \sum_{1 \leq i \leq \frac{1}{2} \log _{2}(x / D)+1}\left(\frac{1}{2^{i-1} \sqrt{D}} \sqrt{x}+1\right) \\
& =O\left(\frac{1}{\sqrt{D}} \sqrt{x}\right) .
\end{aligned}
$$

This completes the proof of Lemma 12.

\section{TheOREM 1.}

$$
\left|A_{x}\right|=\sqrt{\frac{9}{8} x}+o(\sqrt{x}), \quad\left|(\sqrt{2 x}, x] \cap A_{x}\right|=o(\sqrt{x}) .
$$

Pro of. By Lemmas 10, 11 and 8 we have

$$
\begin{aligned}
\sum_{-T \leq i \leq L-1} q^{i}(q-1) \alpha_{i}+(1+\alpha) \sum_{L \leq i \leq T} q^{t}(q-1) \alpha_{i} \\
=\sum_{-T \leq i \leq T} \beta_{i} \alpha_{i} \leq \sum_{-T \leq j \leq L} \delta_{j}+O\left((\log \log x)^{-1 / 2}\right) \\
\leq \sqrt{\frac{9}{8}}-q^{-T}+1-q^{-1}+O\left((\log \log x)^{-1 / 2}\right),
\end{aligned}
$$

where $\alpha=(10-7 \sqrt{2}) / 32$. Hence

$$
\begin{aligned}
& \left|\left(q^{-T} \sqrt{x}, \sqrt{2 x}\right] \cap A_{x}\right|+(1+\alpha)\left|\left(\sqrt{2 x}, q^{T+1} \sqrt{x}\right] \cap A_{x}\right| \\
& \leq \sqrt{\frac{9}{8} x}-q^{-T} \sqrt{x}+\left(1-q^{-1}\right) \sqrt{x}+O\left(\sqrt{x}(\log \log x)^{-1 / 2}\right) .
\end{aligned}
$$

So

$$
\begin{aligned}
\left|[1, \sqrt{2 x}] \cap A_{x}\right|+(1 & +\alpha)\left|\left(\sqrt{2 x}, 2^{S} \sqrt{x}\right] \cap A_{x}\right| \\
& \leq \sqrt{\frac{9}{8} x}+\left(1-q^{-1}\right) \sqrt{x}+O_{1}\left(\sqrt{x}(\log \log x)^{-1 / 2}\right),
\end{aligned}
$$

where $O_{1}$ depends only on $L$ and $S$. By Lemma 12 we have

$$
\left|\left(2^{S} \sqrt{x}, x\right] \cap A_{x}\right|=O_{2}\left(\frac{1}{2^{S}} \sqrt{x}\right),
$$


where the $\mathrm{O}_{2}$-constant is absolute. Therefore $\left|[1, \sqrt{2 x}] \cap A_{x}\right|+(1+\alpha)\left|(\sqrt{2 x}, x] \cap A_{x}\right|$

$$
\leq \sqrt{\frac{9}{8} x}+\left(1-q^{-1}\right) \sqrt{x}+O_{1}\left(\sqrt{x}(\log \log x)^{-1 / 2}\right)+O_{2}\left(\frac{1}{2^{S}} \sqrt{x}\right),
$$

where $O_{1}$ is independent of $x$, and $O_{2}$ is independent of $S$ and $x$. Thus

$$
\left|[1, \sqrt{2 x}] \cap A_{x}\right|+(1+\alpha)\left|(\sqrt{2 x}, x] \cap A_{x}\right| \leq \sqrt{\frac{9}{8} x}+o(\sqrt{x}) .
$$

That is,

$$
\left|A_{x}\right|+\alpha\left|(\sqrt{2 x}, x] \cap A_{x}\right| \leq \sqrt{\frac{9}{8} x}+o(\sqrt{x}) .
$$

Since $\left|A_{x}\right| \geq\left|B_{x}\right|=\sqrt{\frac{9}{8} x}+O(1)$, we have

$$
\left|A_{x}\right|=\sqrt{\frac{9}{8} x}+o(\sqrt{x}), \quad\left|(\sqrt{2 x}, x] \cap A_{x}\right|=o(\sqrt{x}) .
$$

This completes the proof of Theorem 1 .

\section{Proof of the Theorem. First we prove}

THEOREM 2.

$$
\left|\left(\sqrt{\frac{1}{2} x}, \sqrt{2 x}\right] \cap A_{x} \cap(2 \mathbb{Z}+1)\right|=o(\sqrt{x}) .
$$

Proof. Let $L$ be an integer and

$$
q=2^{1 /(2 L)}, \quad I_{i}=\left(q^{i}, q^{i+1}\right], \quad-L \leq i \leq L .
$$

Let

$$
\begin{aligned}
\left\{r_{i u}: u=1, \ldots, k_{i} ; i\right. & =-L,-L+1, \ldots, L\}_{j} \\
= & \left\{r_{j, 1}=0, r_{-j, 1}=-1, r_{-j, 2}=1\right\}, \quad-L \leq j \leq-1 .
\end{aligned}
$$

Then by Lemma 3 we have

Let

$$
\alpha_{j}^{(0)}+2 \alpha_{-j}^{(1)} \leq \frac{1}{2}+O\left((\log \log x)^{-1 / 2}\right), \quad-L \leq j \leq-1 .
$$

$$
\begin{aligned}
\left\{r_{i u}: u=1, \ldots, k_{i} ; i=-L,-L+1, \ldots, L\right\}_{j} & \\
& =\left\{r_{-j, 1}=-1, r_{j, 1}=0, r_{j, 2}=1\right\}, \quad 1 \leq j \leq L .
\end{aligned}
$$

Then by Lemma 3 we have

$$
\alpha_{-j}^{(1)}+\alpha_{j}^{(0)}+\alpha_{j}^{(1)} \leq \frac{1}{2}+O\left((\log \log x)^{-1 / 2}\right), \quad 1 \leq j \leq L .
$$

Let

$$
\begin{aligned}
\left\{r_{i u}: u=1, \ldots, k_{i} ; i=-L,-L+1,\right. & \ldots, L\} \\
& =\left\{r_{0,1}=-1, r_{0,2}=0, r_{0,3}=1\right\} .
\end{aligned}
$$

Then we have

$$
\alpha_{0}^{(0)}+2 \alpha_{0}^{(1)} \leq \frac{1}{2}+O\left((\log \log x)^{-1 / 2}\right)
$$


To use Lemma 6 , let

$$
\begin{aligned}
k_{i j}^{(0)} & =0(i \neq j), & k_{j j}^{(0)} & =1, \\
k_{i j}^{(1)} & =0(i \neq j,-j), & k_{j j}^{(1)} & =1(j \geq 1), \\
k_{j j}^{(1)} & =0(j \leq-1), & k_{(-j) j}^{(1)} & =2(j \leq-1), \\
k_{(-j) j}^{(1)} & =1(j \geq 1), & k_{00}^{(1)} & =2, \\
\delta_{j} & =q^{j}(q-1), & \beta_{i}^{(0)} & =q^{i}(q-1), \\
\alpha & =(10-7 \sqrt{2}) / 32, & \beta_{i}^{(1)} & =(1+\alpha) q^{i}(q-1) .
\end{aligned}
$$

Then

$$
\sum_{-L \leq i \leq L}\left(k_{i j}^{(0)} \alpha_{i}^{(0)}+k_{i j}^{(1)} \alpha_{i}^{(1)}\right)= \begin{cases}\alpha_{j}^{(0)}+2 \alpha_{-j}^{(1)} & \text { if }-L \leq j \leq 0, \\ \alpha_{-j}^{(1)}+\alpha_{j}^{(0)}+\alpha_{j}^{(1)} & \text { if } 1 \leq j \leq L .\end{cases}
$$

Hence

$$
\sum_{-L \leq i \leq L}\left(k_{i j}^{(0)} \alpha_{i}^{(0)}+k_{i j}^{(1)} \alpha_{i}^{(1)}\right) \leq \frac{1}{2}+O\left((\log \log x)^{-1 / 2}\right)
$$

Let

$$
g^{(v)}(t)=\sum_{-L \leq i \leq t} \sum_{-L \leq j \leq L} \delta_{j} k_{i j}^{(v)}, \quad v=0,1 .
$$

Then

$$
g^{(0)}(t)=\sum_{i=-L}^{t} \delta_{i} k_{i i}^{(0)}=\sum_{i=-L}^{t} \delta_{i}=\sum_{i=-L}^{t} \beta_{i}^{(0)}, \quad-L \leq t \leq L .
$$

Now we show that

$$
g^{(1)}(t) \geq \sum_{i=-L}^{t} \beta_{i}^{(1)}, \quad-L \leq t \leq L .
$$

For $-L \leq t \leq-1$ we have (note that $q^{L}=\sqrt{2}$ )

$$
\begin{aligned}
g^{(1)}(t)= & \sum_{i=-L}^{t} \delta_{-i} k_{i(-i)}^{(1)}=\sum_{i=-L}^{t} \delta_{-i}=q^{L+1}-q^{-t} \\
= & (1+\alpha)\left(q^{t+1}-q^{-L}\right)+q^{L+1}+(1+\alpha) q^{-L} \\
& -q^{-t}-(1+\alpha) q^{t+1} \\
\geq & (1+\alpha)\left(q^{t+1}-q^{-L}\right)+q^{L+1}+(1+\alpha) q^{-L} \\
& -\max \left\{q^{L}+(1+\alpha) q^{-L+1}, q+1+\alpha\right\} \\
\geq & (1+\alpha)\left(q^{t+1}-q^{-L}\right) \geq \sum_{i=-L}^{t} \beta_{i}^{(1)} .
\end{aligned}
$$


For $0 \leq t \leq L$ we have

$$
\begin{aligned}
g^{(1)}(t)= & \sum_{i=-L}^{0} \delta_{-i} k_{i(-i)}^{(1)}+\sum_{1 \leq i \leq t}\left(\delta_{i} k_{i i}^{(1)}+\delta_{-i} k_{i(-i)}^{(1)}\right) \\
= & \sum_{i=-L}^{-1} \delta_{-i}+2 \delta_{0}+\sum_{1 \leq i \leq t}\left(\delta_{i}+2 \delta_{-i}\right) \\
= & \sum_{i=-L}^{t} \beta_{i}^{(1)}+q^{L+1}+(1+\alpha) q^{-L}-2 q^{-t}-\alpha q^{t+1} \\
\geq & \sum_{i=-L}^{t} \beta_{i}^{(1)}+q^{L+1}+(1+\alpha) q^{-L} \\
& -\max \left\{2+\alpha q, 2 q^{-L}+\alpha q^{L+1}\right\} \\
\geq & \sum_{i=-L}^{t} \beta_{i}^{(1)} .
\end{aligned}
$$

By (14)-(17) and Lemma 6 we have

$$
\begin{aligned}
\sum_{i=-L}^{L} q^{i}(q-1) \alpha_{i}^{(0)}+\sum_{i=-L}^{L} q^{i}(q-1) \alpha_{i}^{(1)}+\alpha \sum_{i=-L}^{L} q^{i}(q-1) \alpha_{i}^{(1)} \\
=\sum_{i=-L}^{L}\left(\beta_{i}^{(0)} \alpha_{i}^{(0)}+\beta_{i}^{(1)} \alpha_{i}^{(1)}\right) \leq \frac{1}{2} \sum_{j=-L}^{L} \delta_{j}+O\left((\log \log x)^{-1 / 2}\right) \\
\leq \frac{1}{4} \sqrt{2}+\frac{1}{2} \sqrt{2}(q-1)+O\left((\log \log x)^{-1 / 2}\right) .
\end{aligned}
$$

Hence

$$
\begin{aligned}
\left|\left(q^{-L}, q^{L+1}\right] \sqrt{x} \cap A_{x}\right| & +\alpha\left|\left(q^{-L}, q^{L+1}\right] \sqrt{x} \cap A_{x} \cap(2 \mathbb{Z}+1)\right| \\
\leq & \frac{1}{4} \sqrt{2 x}+\frac{1}{2} \sqrt{2 x}(q-1)+O\left(\sqrt{x}(\log \log x)^{-1 / 2}\right) .
\end{aligned}
$$

It is clear that

$$
\left|\left[1, q^{-L}\right] \sqrt{x} \cap A_{x}\right| \leq \frac{1}{2} \sqrt{2 x}
$$

So

$$
\begin{aligned}
\left|[1, \sqrt{2 x}] \cap A_{x}\right|+\alpha \mid & \left(\sqrt{\frac{1}{2} x}, \sqrt{2 x}\right] \cap A_{x} \cap(2 \mathbb{Z}+1) \mid \\
& \leq \sqrt{\frac{9}{8} x}+\frac{1}{2} \sqrt{2 x}(q-1)+O\left(\sqrt{x}(\log \log x)^{-1 / 2}\right) .
\end{aligned}
$$

Hence

(18) $\left|[1, \sqrt{2 x}] \cap A_{x}\right|+\alpha\left|\left(\sqrt{\frac{1}{2} x}, \sqrt{2 x}\right] \cap A_{x} \cap(2 \mathbb{Z}+1)\right| \leq \sqrt{\frac{9}{8} x}+o(\sqrt{x})$. 
By Theorem 1 we have

$$
\left|[1, \sqrt{2 x}] \cap A_{x}\right|=\sqrt{\frac{9}{8} x}+o(\sqrt{x}) .
$$

Thus by (18) we have

$$
\left|\left(\sqrt{\frac{1}{2} x}, \sqrt{2 x}\right] \cap A_{x} \cap(2 \mathbb{Z}+1)\right|=o(\sqrt{x}) .
$$

This completes the proof of Theorem 2.

Proof of the Theorem. By Theorem 1 we have

$$
\left|A_{x}\right|=\left|B_{x}\right|+o(\sqrt{x})=\sqrt{\frac{9}{8} x}+o(\sqrt{x}) .
$$

By Theorems 1 and 2 we have

$$
\left|A_{x} \backslash B_{x}\right| \leq\left|A_{x} \cap\left[\sqrt{\frac{1}{2} x}, \sqrt{2 x}\right] \cap(2 \mathbb{Z}+1)\right|+\left|A_{x} \cap(\sqrt{2 x}, x]\right|=o(\sqrt{x}) .
$$

This completes the proof of the Theorem.

Acknowledgements. I am very grateful to Professor A. Schinzel for carefully reading my first proof of Theorem 1 and giving me some suggestions.

\section{References}

[1] S. L. G. Choi, The largest subset in $[1, n]$ whose integers have pairwise l.c.m. not exceeding n, Mathematika 19 (1972), 221-230.

[2] - The largest subset in $[1, n]$ whose integers have pairwise l.c.m. not exceeding $n$, II, Acta Arith. 29 (1976), 105-111.

[3] P. Erdős, Problem, Mat. Lapok 2 (1951), 233.

[4] -, Extremal problems in number theory, in: Theory of Numbers, Proc. Sympos. Pure Math. 8, Amer. Math. Soc., 1965, 181-189.

[5] R. K. Guy, Unsolved Problems in Number Theory, 2nd ed., Springer, New York, 1994.

[6] H. Halberstam and H. E. Richert, Sieve Methods, Academic Press, London, 1974.

Department of Mathematics

Nanjing Normal University

Nanjing 210097, China

E-mail: ygchen@pine.njnu.edu.cn

Received on 7.10 .1997

and in revised form on 10.11.1997 